\title{
Análise com assistentes virtuais inteligentes: Um estudo de caso com o Google Assistente
}

\author{
Daiane Rose da Silva Matos ${ }^{1}$, Francisco Kelsen de Oliveira ${ }^{2}$ \\ ${ }^{1,2}$ Instituto Federal de Educação, Ciência e Tecnologia do Sertão Pernambucano \\ (IFSertãoPE) - Salgueiro-PE-Brasil \\ https://orcid.org/0000-0002-9376-6464'; https://orcid.org/0000-0002-7382-3206²; \\ \{daianersm2@gmail.com ${ }^{1}$, francisco.oliveira@ifsertao-pe.edu.br ${ }^{2}$ \}
}

\begin{abstract}
Resumo. Este artigo objetivou analisar a experiência dos usuários com o assistente virtual presente em smartphones que dispõem do Sistema Operacional (S.O) Android em tarefas peculiares do uso discente. A pesquisa contemplou alunos do curso Superior de Tecnologia em Sistemas para Internet do IFSertãoPE, buscando compreender se tais ferramentas conseguem auxiliar os estudantes na realização de suas atividades enquanto discentes. Os resultados alcançados identificaram ainda dificuldades dos usuários em realizar as tarefas pela assistente virtual de voz, embora sejam tarefas comumente realizadas por todos a partir de outras formas de interações. Palavras-chave: Assistente de voz; Tecnologia de suporte ao Ensino; Aprendizagem móvel.
\end{abstract}

\section{Analysis with Smart Virtual Assistants: A Case Study with Google Assistant}

\begin{abstract}
This paper aimed to analyze the experience of users with the virtual assistant present in smartphones that have the Android Operating System (OS) in peculiar tasks of student use. The research included students of the Higher Course of Technology in Internet Systems of IFSertãoPE, seeking to understand if these tools can help students in performing their activities as students. The results also identified difficulties for users in performing tasks by the virtual voice assistant, although they are tasks commonly performed by everyone from other forms of interactions.
\end{abstract}

Keywords: Voice assistant; Teaching support technology; Mobile learning.

\section{Introdução}

$\mathrm{O}=$ surgimento $=\mathrm{do}=\mathrm{computador}=\mathrm{e}=\log \mathrm{o}=\mathrm{e} \mathrm{m}=$ seguida $=\mathrm{sua}=\mathrm{conexão}=\mathrm{a}=$ internet possibilitou $=\mathrm{a}=$ propagação $=\mathrm{de}=$ conteúdos $=$ midiáticos $=\mathrm{e}=0=\mathrm{compartilhamento}=\mathrm{de}=$ informações $=$ diversas,$=$

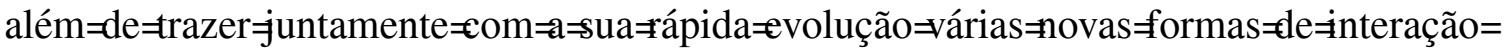

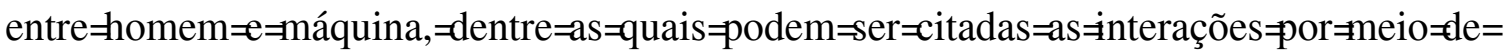
interfaces $=$ gestuais, $=$ realidade $=$ aumentada, $=$ realidade $=v i r t u a l,=$ realidade $=$ mista $=($ MAUÉS,$=$ 2019),$=$ como $=$ também $=$ atravé $=$ das $=$ populares $=$ Interfaces $=\mathrm{de}=$ Usuário $=\mathrm{de}=\mathrm{Voz}=($ do=inglês $=$ Voice User Interfaces,$=$ VUIs $),=\mathrm{que},=\mathrm{em}=\mathrm{geral},=\mathrm{utilizam}=\mathrm{comandos}=\mathrm{de}=\mathrm{voz}=\mathrm{ou}=\mathrm{fala}=\mathrm{para}=$ permitir $=q u e=0 \mathrm{~s}=$ usuários=interajam $=\mathrm{com}=u \mathrm{~m}=\mathrm{determinado}=\mathrm{sistema}=\mathrm{ou}=\mathrm{serviço}=\mathrm{de}=$ forma 
fácil, rápida e divertida, sem exigir que o usuário esteja necessariamente segurando ou olhando diretamente para o dispositivo.

Assistentes virtuais como Alexa, Siri e o Google Assistant são considerados exemplos de VUIs, agentes portadores de Inteligência Artificial (IA) capazes de realizar diversas tarefas dentro de um sistema. A interação com esses assistentes tem a intenção de requerer o mínimo de esforço cognitivo possível por parte dos usuários, tornando simples a execução de inúmeras tarefas do cotidiano como o envio de mensagens de texto, buscas online e organização de agendas, podendo ser facilmente encontrados em grandes Sistemas Operacionais (SO) de smartphones, a exemplo destacam-se o Android da Google e o iOS da Apple.

Tais assistentes tiveram um grande aumento do seu alcance devido estarem presentes em diferentes tipos de celulares ou dispositivos de baixo custo largamente utilizados nos dias de hoje. Ao longo dos anos, a utilização de dispositivos móveis, em especial os smartphones, tem sido cada vez maior e vem adquirindo grande popularidade dentro da sociedade contemporânea. Segundo a Pesquisa Nacional por Amostra de Domicílios - PNAD (IBGE, 2020), constatou-se que em 2018 o telefone móvel celular era o dispositivo mais utilizado para conexão com à internet, atingindo 99,2\% dos domicílios em área urbana e 99,4\% em área rural, alcançando dessa maneira quase a totalidade dos domicílios. Em contrapartida, desde o ano de 2017 nota-se que a utilização de microcomputadores $\mathrm{v}$ em sofrendo um decl ínio contínuo e e vidente.

Possíveis justificativas para tamanha popularidade seriam: mobilidade e portabilidade oferecidas por esses aparelhos possibilitando aos usuários levá-lo a qualquer lugar; aspectos cognitivos permitindo a interação com diversos recursos em vários formatos como sons, textos, imagens e vídeos; e a conectividade por meio da internet no celular, ampliando as formas de comunicação e tornando viável o acesso à múltiplos tipos de interações e informações (FONSECA, 2013).

Contudo, apesar de tantos benefícios, para que a utilização de tais tecnologias seja realmente proveitosa é necessário que sejam bem projetadas e apresentem uma boa usabilidade, isto é, assegurem que uma pessoa utilize determinada interface para realizar uma tarefa específica com facilidade (CYBIS, BETIOL, FAUST 2015). Esta ideia se encontra em conformidade com a norma NBR 9241-11 (ABNT/NBR, 2002) que conceitua a usabilidade como o quanto um produto pode ser usado por um usuário específico para atingir um objetivo específico com eficiência, eficácia e satisfação em um determinado conte xto de uso.

A Experiência do Usuário (Do inglês User eXperience - UX) apresenta-se nesse cenário com um sentido mais amplo, englobando não apenas características e metas objetivas da usabilidade, mas toda a experiência proporcionada aos usuários, focada em aspectos subjetivos como o quanto uma aplicação é cativante, divertida e agradável de ser usada (LO WDERMILK, 2013).

Portanto, este trabalho objetivou analisar a experiência dos usuários com o assistente virtual presente em smartphones que dispõem do S.O Android em tarefas peculiares do uso discente. Levando em consideração o contexto do Ensino Remoto Emergencial (ERE), procurou-se compreender se os estudantes conseguiam realizar suas atividades discentes a partir do dispositivo móvel e com o auxílio do assistente utilizando a voz como interface. Assim, buscamos responder aos seguintes questionamentos: Quais os impactos provenientes das interações desses usuários com 
esses dispositivos com vistas às suas atividades discentes? Os usuários conhecem as funcionalidades disponíveis em seus dispositivos e como usá-los?

$\mathrm{O}$ artigo é formado por seis seções, conforme a seguinte ordem: referenciais teóricos, metodologia aplicada à pesquisa, resultados e discussões alcançados, as considerações finais e as referências bibliográficas que embasaram a pesquisa.

\section{Referencial Teórico}

Assistentes virtuais inteligentes, também conhecidos como assistentes pessoais virtuais (Do inglês Virtual Personal Assistants - VPAs) são softwares projetados para interagir com diversos tipos de usuários em linguagem natural (CRUZ et al., 2013) e utilizar os seus principais métodos de inputs (entrada) de dados comandos de voz ou texto. Tais softwares são um meio de ajudar as pessoas a executarem uma grande quantidade de tarefas específicas do seu cotidiano, seja no trabalho, nos estudos ou para o entretenimento, desde realizar busca de documentos e informações online, enviar e-mails até executar tarefas um pouco mais avançadas como monitorar e comandar residências e aparelhos domésticos, tornando-as prática e asserti vas.

De acordo com a abordagem de Falavigna (2015), a interação que ocorre entre as pessoas e os computadores mediada por uma interface é um estudo proveniente de algumas áreas da Ciência da Computação, especificamente do campo da Interação Humano-Computador (IHC). O interesse da IHC está na totalidade da interação entre o usuário e a máquina envolvendo o desenvolvimento das interfaces, a implementação, a qualidade de uso dos sistemas interativos e de que maneira os meios computacionais afetam os usuários.

Dentre os atributos de qualidade imprescindíveis da IHC, encontra-se a Usabilidade, que assegura o quão eficiente, fácil e agradável de usar é um sistema a partir da perspectiva do usuário (ROGERS; SHARP; PREECE, 2013). O fato de sistemas interativos estarem presentes apenas em ambientes de trabalho até pouco tempo e, muitas vezes, limitava o foco das investigações aos aspectos ligados à eficiência e eficácia atingidas pelo usuário na realização de um tarefa, porém com a expansão do uso de tais meios interativos para outros contextos como educação, saúde, comércio, entretenimento nos diversos âmbitos da sociedade, então se fez necessário compreender também os aspectos subjetivos ligados à maneira com que o uso de um sistema afeta os sentimentos, emoções e percepções dos usuários, estendendo dessa forma a atenção nitidamente restrita da usabilidade para a experiência de uso como um todo.

A Experiência do Usuário está relacionada à totalidade da interação vivenciada entre o usuário e um determinado produto, serviço ou sistema, desde a concepção do interesse ou necessidade em adquiri-lo à execução propriamente dita de tal interação e posteriormente sua reflexão sobre o momento vivenciado. O termo UX foi popularizado pelo psicólogo cognitivo e pesquisador Donald Norman na década de 90 e engloba aspectos referentes a como as pessoas se sentem em relação a um produto, ao prazer, e à satisfação obtidos ao percebê-lo, olhá-lo ou usá-lo (ROGERS; SHARP; PREECE, 2013).

Para Rogers, Sharp e Preece (2013), as metas da experiência do usuário diferem-se das metas de usabilidade por buscarem explorar como o utilizador 
experimenta um produto interativo sob sua perspectiva em vez de avaliar se o mesmo é útil e produtivo sob a perspectiva do próprio sistema. Essas metas cobrem uma diversidade de experiências emocionais e sensoriais, incluindo experiências agradáveis e desagradáveis. Pensar na experiência que o usuário está vivenciando é lidar muitas vezes com cenários incertos e difíceis de ser mensurado pois diversos são os fatores que influenciam a percepção do usuário e a maneira que ele constrói suas expectativas e expõe suas experiências.

Ainda segundo as observações de Petrie e Bevan (2009), UX é o termo mais recente no conjunto de critérios em relação aos quais um sistema deve ser avaliado e continuará tendo grande importância futuramente, isso porque ao se tornarem ubíquos no cotidiano das pessoas os usuários esperam mais do que um sistema fácil de usar. Portanto a experiência do usuário surge para cobrir componentes das interações dos usuários e suas reações ao sistema que vão além da eficiência, eficácia e análises convencionais da satisfação, permitindo o desenvolvimento de um entendimento emocional maior do usuário com o sistema e o que tal relação significa para os usuários.

\section{Metodologia}

A pesquisa adotou uma abordagem de natureza quantitativa e qualitativa uma vez que buscou como objeto de estudo avaliar a experiência dos usuários estudantes do curso Superior de Tecnologia em Sistemas para Internet com o assistente virtual Google Assistant, presente em smartphones que dispõem do Android OS, utilizando a voz como interface. Quanto ao caráter, é considerado descritivo e exploratório, pois buscou conhecer, interpretar e detalhar o comportamento dos usuários diante das tarefas propostas. (APPOLINÁRIO, 2012).

Considerando o fundamento da pesquisa de analisar a experiência dos estudantes com o assistente virtual no que diz respeito à realização das suas atividades rotineiras enquanto discentes, buscando identificar possíveis problemas e propor melhorias, foram elaboradas para o experimento e submetidas aos participantes nove (9) tarefas sequencialmente elencadas no Quadro 1.

Quadro 1: Tarefas submetidas aos estudantes.

\begin{tabular}{|l|l|l|}
\hline ID & \multicolumn{1}{|c|}{ Título } & \multicolumn{1}{|c|}{ Objetivo } \\
\hline T1 & $\begin{array}{l}\text { Busque na Internet por artigos sobre } \\
\text { Interação Humano-Computador } \\
\text { (IHC) e salve no seu dispositivo }\end{array}$ & $\begin{array}{l}\text { Verificar se o estudante a partir de comandos de } \\
\text { voz conseguiria acessar arquivos específicos na } \\
\text { internet e se esses poderiam ser salvos em seu } \\
\text { dispositivo. }\end{array}$ \\
\hline T2 & $\begin{array}{l}\text { Localize a pasta que o arquivo do } \\
\text { item 1 foi salvo }\end{array}$ & $\begin{array}{l}\text { Observar se o assistente virtual acionado por } \\
\text { comandos de voz conseguiria retornar ao } \\
\text { estudante o local onde o arquivo baixado havia } \\
\text { sido salvo. }\end{array}$ \\
\hline T3 & $\begin{array}{l}\text { Abra o arquivo do item 2 pelo } \\
\text { aplicativo que permita a leitura por } \\
\text { voz do assistente virtual a partir do }\end{array}$ & $\begin{array}{l}\text { Verificar se o assistente virtual conseguiria } \\
\text { realizar a leitura em voz alta de um arquivo, } \\
\text { tendo em vista que por exemplo muitos }\end{array}$ \\
\hline
\end{tabular}




\begin{tabular}{|c|c|c|}
\hline & $\begin{array}{l}\text { texto apresentado no arquivo. (Obs: } \\
\text { tenha um aplicativo que realize a } \\
\text { leitura de pdf por voz) }\end{array}$ & $\begin{array}{l}\text { estudantes poderiam utilizar tal ferramenta } \\
\text { como auxílio de leitura no percurso de casa para } \\
\text { à faculdade ou em outra situação. }\end{array}$ \\
\hline $\mathrm{T} 4$ & $\begin{array}{l}\text { Abra o aplicativo de edição de texto } \\
\text { Google Docs ou similar }\end{array}$ & $\begin{array}{l}\text { Averiguar se o estudante por meio da interação } \\
\text { por voz conseguiria ter acesso rápido à alguma } \\
\text { ferramenta de edição de texto. }\end{array}$ \\
\hline T5 & $\begin{array}{l}\text { Crie um documento contendo a frase } \\
\text { "aprendendo usabilidade na prática" }\end{array}$ & $\begin{array}{l}\text { Averiguar se o estudante a partir do assistente } \\
\text { virtual conseguiria realizar a escrita de uma } \\
\text { frase em um documento de texto. }\end{array}$ \\
\hline T6 & $\begin{array}{l}\text { Salve um arquivo com o nome } \\
\text { "ResumoIHC" }\end{array}$ & $\begin{array}{l}\text { Verificar se através do assistente virtual e } \\
\text { utilizando comandos de voz o estudante } \\
\text { conseguiria nomear e salvar um arquivo de } \\
\text { texto. }\end{array}$ \\
\hline $\mathrm{T} 7$ & $\begin{array}{l}\text { Envie um e-mail para o } \\
\text { francisco.oliveira@ ifsertao-pe.edu.br } \\
\text { perguntando se haverá aula de IHC. }\end{array}$ & $\begin{array}{l}\text { Observar se a partir do assistente virtual o } \\
\text { estudante conseguiria enviar um e-mail a um } \\
\text { professor da Instituição com o objetivo de sanar } \\
\text { alguma dúvida. }\end{array}$ \\
\hline T8 & $\begin{array}{l}\text { Participe da aula da disciplina de } \\
\text { IHC no Meet, pelo link: } \\
\text { https://meet.google.com/lookup/ahh } \\
\text { ux3bh3g?authuser=1\&hs=179 }\end{array}$ & $\begin{array}{l}\text { Compreender se é possível a partir do assistente } \\
\text { virtual e utilizando comandos de voz o estudante } \\
\text { participar de uma reunião disponível na } \\
\text { ferramenta Google Meet. }\end{array}$ \\
\hline T9 & $\begin{array}{l}\text { Participe da turma de IHC disponível } \\
\text { no Google Classroom através do } \\
\text { código ( } 2 \text { f2e } 561)\end{array}$ & $\begin{array}{l}\text { Compreender se é possível a partir do assistente } \\
\text { virtual e utilizando comandos de voz o estudante } \\
\text { participar de uma turma disponível na } \\
\text { ferramenta Google Classroom. }\end{array}$ \\
\hline
\end{tabular}

Fonte: Pesquisa direta.

O perfil selecionado para integrar o grupo amostral foi de alunos regularmente matriculados no curso Superior de Tecnologia em Sistemas para Internet (SPI) do campus Salgueiro do IFSertãoPE. A amostra contou com a participação de dez (10) estudantes escolhidos de forma aleatória, podendo ser identificados no grupo, alunos do $3^{\circ}$ (terceiro), $5^{\circ}$ (quinto) e $6^{\circ}$ (se xto) período d mespectivo curso.

A fase da coleta dos dados foi realizada de forma inteiramente remota, utilizando a ferramenta Google Meet como ambiente para reunião e consequentemente execução das tarefas com os estudantes. Inicialmente os participantes foram orientados quanto à autorização prévia e necessidade do compartilhamento da tela dos seus smartphones para que fosse possível aos pesquisadores observar toda a interação com o assistente, para isso foram instruídos a realizarem algumas tarefas relacionadas à devida instalação e configuração dos seus dispositi vos.

Todas as tarefas executadas pelos participantes foram registradas em vídeo para posterior análise, utilizando a ferramenta de gravação do próprio Google Meet e a ferramenta online e gratuita Apowersoft. Antes da realização do experimento, os alunos 
de forma individual foram devidamente orientados quanto aos procedimentos e etapas que deveriam seguir, receberam antecipadamente a lista das tarefas que deveriam executar para que pudessem ir se familiarizando, como também receberam o auxílio dos pesquisadores na leitura das mesmas. Foram orientados ainda sobre a necessidade de realizarem toda a interação com o assistente somente por comandos de voz.

Os dados coletados no experimento foram tabulados e organizados em planilha eletrônica com o objetivo de facilitar a análise adequada de variáveis específicas. Foram consideradas as seguintes variáveis: Tempo de Execução (TE) padronizado em segundos, tendo o objetivo de mensurar por exemplo se o usuário sentiu-se confuso com o que deveria ser executado ou se já possuía alguma experiência com determinada tarefa; Número de Tentativas (NT) buscando aferir se por exemplo o usuário estava adotando uma metodologia de tentar várias sequências de possibilidades até encontrar uma que realmente funcionasse ou não; e o Estado da Tarefa (ES) visando estabelecer se o participante concluiu, não concluiu ou realizou parcialmente a tarefa e se esses resultados estavam relacionados a um possível conhecimento prévio da ferramenta ou se o sistema era intuitivo de utilizar .

$\mathrm{Na}$ seção subsequente serão apresentados os resultados obtidos dos experimentos.

\section{Resultados e discussões}

De acordo com os dados fornecidos pelos participantes da pesquisa $40 \%$ cursavam o terceiro semestre, $30 \%$ o quinto semestre e $30 \%$ o sexto (último) semestre do curso de Tecnologia em Sistemas para Internet no campus Salgueiro do IFSertãoPE, sendo 80\% alunos do sexo masculino e $20 \%$ do sexo feminino, vale ressaltar que $60 \%$ dos integrantes cursam ou já cursaram a disciplina de Interação Humano-Computador (IHC).

Através da análise dos resultados foi possível notar na $T 1$, que tratava sobre buscar e salvar artigos da internet, que $100 \%$ dos participantes conseguiram realizar a tarefa parcialmente. Percebeu-se que o sucesso ocorreu no momento em que os estudantes solicitaram a busca dos artigos, alcançando o retorno rapidamente. Já o insucesso ocorreu quando os alunos solicitaram ao assistente que um dos artigos fosse salvo no dispositivo, o assistente perdia o contexto do diálogo fazendo com que $50 \%$ dos alunos demandassem uma média de três (3) minutos na execução da tarefa em razão da dificuldade de encontrar o comando de voz adequado e tentando refazer o caminho em busca de corrigir o problema. $60 \%$ dos alunos relataram durante a interação que saberiam executar facilmente o download se usassem a interação manual com o dispositivo.

$\mathrm{Na} \mathrm{T} 2$, localizar pasta de arquivo salvo, $60 \%$ dos estudantes não conseguiram concluir a tarefa e $40 \%$ realizaram parcialmente. Em virtude de não obterem êxito no download do artigo da T1, 50\% dos alunos decidiram não realizá-la ou não ultrapassar mais do que 1 tentativa, outros se mostraram bastante confusos por não saberem os comandos adequados que deveriam utilizar para que o assistente executasse tal ação, resultando o método tentativa e erro. Os que conseguiram realizá-la de forma parcial mostraram possuir um certo conhecimento em relação aos comandos que deveriam seguir chegando até a etapa de abrir a pasta de arquivos e downloads do dispositivo, 
porém como esta tarefa estava ligada ao sucesso da T1, o arquivo não havia sido salvo e a taref a foi considerada incompleta.

A T3 que solicitava a leitura por voz de um arquivo através da assistente virtual não foi concluída por nenhum dos participantes. $20 \%$ dos alunos decidiram não executar a tarefa e $30 \%$ não ultrapassaram mais do que uma (1) tentativa já que o enunciado associava a tarefa ao sucesso da T1 e da T2 e preferiram avançar para as próximas tarefas. Foi possível perceber ainda que $40 \%$ dos alunos que continuaram tentando realizar a tarefa proposta optaram pelo método tentativa e erro, testando várias possibilidades como forma de chegar ao resultado esperado exigindo assim uma permanência maior na e xecução da taref a.

A tarefa que requisitava a abertura de um aplicativo de edição de texto identificada como $T 4$ foi realizada com sucesso pela maioria dos participantes, $90 \%$ dos alunos obtiveram êxito em uma média de tempo geral de 36 segundos e utilizando em média uma (1) tentativa para alcançar o objetivo. Isso se dá pelo fato da tarefa ser simples, intuiti va e não e xigir v árias etapas a sere seguidas.

Notou-se que todos os participantes apresentaram dificuldades para realizar a $T 5$ que sugeria criar um documento contendo uma frase, resultando em $100 \%$ dos estudantes não concluindo a tarefa. Foi possível perceber que durante a interação muitos se mostraram indecisos e pensativos sobre quais comandos utilizar para que o assistente compreendesse o que deveria ser feito, resultando no método de tentativa e erro e demandando uma média de dois (2) minutos na execução da tarefa. Viu-se que alguns alunos já estavam apresentando um certo desânimo em tentar realizar a tarefa citando frases como: "Eu desisto, ela não me entende mesmo" e "É, acredito que não consigo executar essa taref a também".

Na tarefa identificada como T6, 100\% dos alunos apresentaram dificuldades e não conseguiram chegar à conclusão. Foi possível perceber que em razão da falta de sucesso relacionada à algumas tarefas passadas que já haviam solicitado ações semelhantes, $70 \%$ dos estudantes optaram por não realizar mais do que três (3) tentativas e decidiram se guir para as próximas taref as.

Acerca da $T 7$ onde o estudante deveria enviar um e-mail, $30 \%$ dos participantes realizaram a tarefa parcialmente e $70 \%$ não conseguiu concluí-la. Pôde-se observar que os alunos encontraram dificuldades no momento de fornecer o e-mail do destinatário, aos que já possuíam de certa forma o contato previamente armazenado este momento exato não foi tão complicado, porém aos que não possuíam, a interação apenas por voz foi quase que impossível de ser realizada. Além disso, em várias ocasiões o assistente virtual não compreendeu corretamente a mensagem que deveria ser enviada, isso fez com que os alunos repetissem a sequência várias vezes, atingindo uma média de 8 tentativas demandando assim muito tempo na e xecução da taref a.

A respeito da $T 8$ que solicitava participar de uma reunião na plataforma Google Meet, $50 \%$ dos estudantes realizaram a tarefa de forma parcial e 50\% não conseguiram concluí-la. Notou-se que alguns participantes tinham facilidade em abrir o aplicativo por meio de comandos de voz, porém não conseguiam avançar além disso. Percebeu-se ainda que vários alunos ficaram confusos e indagaram a respeito do enunciado da tarefa por não saberem de que forma forneceriam ao assistente o link disponibilizado, resultando em um tempo elevado na execução da tarefa. Além disso, em quase todas as interações, frequentemente o assistente compreendia e executava a aplicação "Google 
Maps" em lugar de "Google Meet", ocasionando uma repetição contínua do mesmo comando por parte dos usuários para corrigir o equívoco.

A T9 que requisitava a participação em uma turma no Google Classroom mostrou que $80 \%$ dos alunos realizaram a tarefa parcialmente, enquanto $20 \%$ não conseguiram concluí-la. Os participantes encontravam facilidade em pedir ao assistente para abrir a aplicação solicitada, porém apresentaram dificuldades ao solicitar o ingresso em uma turma específica, demandando muito tempo na execução buscando a melhor forma de fazer o assistente compreender o que deveria ser feito. Alguns alunos informaram que inserindo o código de forma manual conseguiriam completar a tarefa com maior facilidade.

Comum à todas as tarefas, a maior parte dos estudantes informaram que conseguiriam facilmente concluí-las se pudessem, além de comandos de voz, interagir também de forma manual com o dispositivo. Verificou-se ainda que em todas as tarefas o assistente frequentemente não compreendia, confundia e executava de forma incorreta os comandos e a linguagem utilizada pelos estudantes fazendo com que fosse necessário refazer a sequência várias vezes no intuito de corrigir o erro. Outra reclamação comum foi em relação ao assistente perder constantemente informações anteriores e o contexto do diálogo, fazendo parecer que todo comando ou frase pronunciada tratava-se de uma nova conversa. Além disso, pôde-se perceber que por não disponibilizar funções de conversa ou diálogo contínuo na sua versão em português brasileiro, muitos alunos demonstraram desconforto em ter que repetir a todo momento a frase de invocação "Ok Google" para acionar o assistente.

Nesse sentido, como mostra o gráfico 1, pôde-se concluir que tarefas que exigiam ações complexas como realizar downloads, enviar e-mails, criar, salvar, abrir e renomear arquivos, manter diálogos com contextos contínuos, bem presentes na T3, T5, T6 e T7 foram difíceis de ser executadas atingindo altos índices de não sucesso das tarefas por parte dos participantes se comparado à ações mais simples como realizar buscas online e abrir aplicações pré-instaladas no dispositivo encontradas, na T1, T4.

\section{Gráfico 1. Estado das tarefas executadas.}

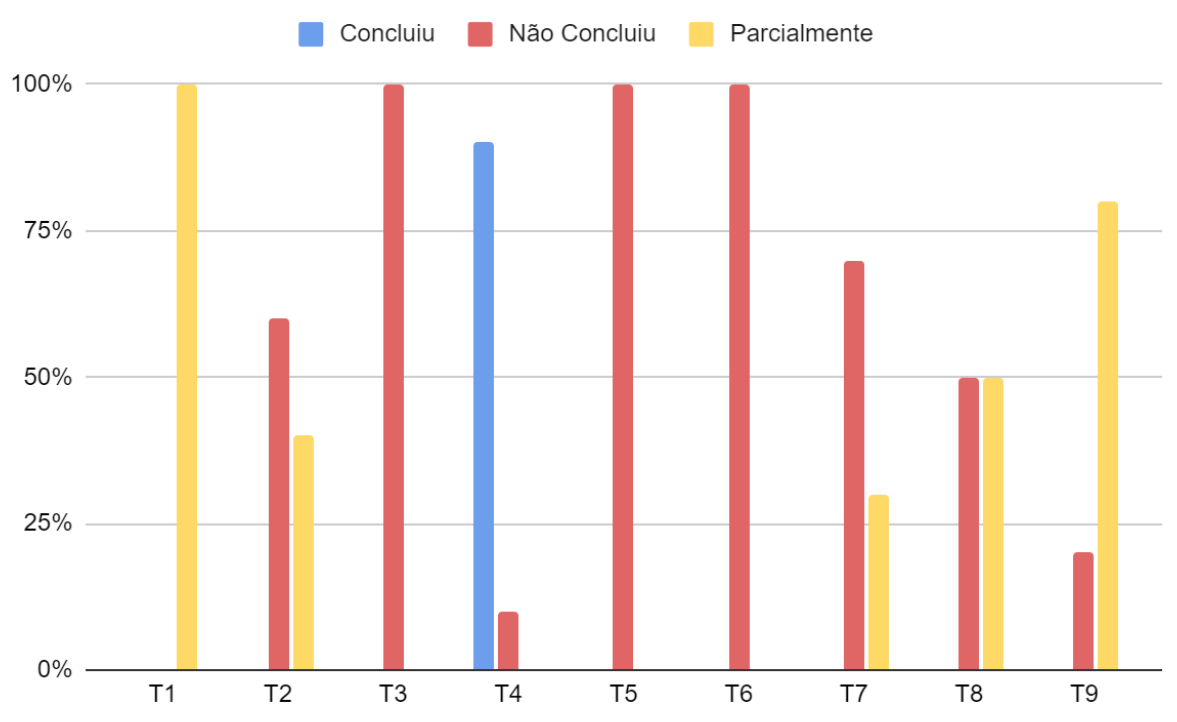

Fonte: Pesquisa direta. 
Dessa forma, percebe-se que tarefas voltadas para realização de uma tarefa direta ou que não exija a produção de conteúdo e sim o consumo direto pode ser realizado de forma mais fácil pelos usuários, tal qual como ocorreu no caso de abrir uma aplicação. Enquanto outras atividades que necessitavam produzir um pequeno texto se tornava mais difícil, muitas vezes, causado pela própria fala rápida ou com vícios de linguagem característicos da linguagem oral informal com reduções de sílabas finais ou mesmo de fonemas que geravam outras palavras diferentes daquelas a serem realmente mencionadas. Portanto, percebe-se que algumas dessas tarefas poderiam ter sido concluídas com uso de termos adequados, enquanto outras com preenchimentos de campos diferentes como o caso do e-mail são mais difíceis, principalmente, nas passagens de um campo para outro.

\section{Considerações finais}

A premissa desta pesquisa tratou sobre a interação por voz entre estudantes da área de informática e o assistente virtual Google Assistant presente em smartphones, acreditando que tal ferramenta traria impactos positivos e auxiliaria os usuários na realização das suas ati vidades enquanto discentes.

Ao final da investigação, compreendeu-se que embora possua muitas funcionalidades e suporte a tarefas variadas no dia a dia das pessoas, os participantes da amostra não conseguiram utilizar tal assistente de forma eficaz para realizar tarefas específicas, bem como relataram que muitas dessas tarefas são utilizadas por outras formas de interações. O uso do assistente virtual para execução de tais tarefas demonstrou ter impactado de forma negativa e gerado uma experiência desconfortável para os usuários. Demonstrou ainda que muitos alunos possuem um certo conhecimento e utilizam a ferramenta para diversas finalidades, porém com o auxílio da interação manual para real izar determinadas ações no sistema.

Diante disso, espera-se que os resultados obtidos possam servir de base para pesquisas futuras e motivação para o desenvolvimento de interfaces voltadas para auxiliar usuários a realizar suas atividades no contexto estudantil, buscando minimizar os problemas aqui apresentados e proporcionar uma experiência de uso agradável e completa. Como a pesquisa restringiu-se apenas à observar a interação com o Google Assistant por apresentar maior disponibilidade entre os participantes, pode-se estender o estudo e aplicar a metodologia a outros assistentes presentes em outros sistemas operacionais, bem como a outros conte xtos para pesquisa futuras.

Além disso, pode haver um maior esforço por parte dos mantenedores e desenvolvedores do software para aproximar a interação de recursos básicos com termos em idioma português para facilitar o uso do recurso, bem como a divulgação dessas formas pelos usuários, tal qual a Google tem realizado com envio de e-mails que apresentam as expressões a serem utilizadas para tarefas de ouvir música, abrir e-mail, etc.

\section{Referências}


Appolinário, F. (2012) "Metodologia da ciência: filosofia e prática da pesquisa". Cengage Learning, São Paulo/SP.

ASSOCIAÇÃO BRASILEIRA DE NORMAS TÉCNICAS. NBR 9241: requisitos ergonômicos para trabalho de escritórios com computadores. Rio de Janeiro, 2002.

Cruz, L. T.; Alencar, A. J., Schmitz, E. A. (2013) "Assistentes Virtuais Inteligentes: Conceitos e Estratégias". Rio de Janeiro: Brasport.

Cybis, W.; Betiol, A.; Faust, R. (2015) "Ergonomia e Usabilidade $3^{\text {a }}$ edição: Conhecimentos, Métodos e Aplicações". Novatec Editora.

Falavigna, V. D. (2015), "Experiência do Usuário: Análise e Aplicação de Métodos de Avaliação". Dissertação de Mestrado, Universidade de Caxias do Sul.

Fonseca A. G. M. F. (2013) "Aprendizagem, Mobilidade e Convergência: Mobile Learning com Celulares e Smartphones". Revista Eletrônica do Programa de Pós-Graduação em Mídia e Cotidiano. Artigos Seção Livre. Número 2. 163-181.

IBGE. Pesquisa Nacional por Amostra de Domicílios Contínua. (2020) "Acesso à internet e à televisão e posse de telefone móvel celular para uso pessoal 2018”. Instituto Brasileiro de Geografia e Estatística (IBGE). Rio de Janeiro.

Lowdermilk, T. (2013) "Design Centrado no Usuário: Um guia para o desenvolvimento de aplicativos amigáveis". São Paulo: Novatec Editora.

Maués, M. P. (2020) "Um olhar sobre os assistentes de voz personificados e a voz como interface”. Dissertação (Mestrado em design), Departamento de Artes e Design, Pontifícia Universidade Católica do Rio de Janeiro, Rio de Janeiro.

Petrie, H.; Bevan, N. (2009) "The Evaluation of Accessibility, Usability, and User Experience". The universal access handbook, v. 1, p. 1-16.

Rogers, Y.; Sharp, H.; Preece, J. (2013) "Design de Interação: além da interação humano-computador". 3. ed. Porto Alegre: Bookman. 